\title{
Peritoneal dialysis catheters in pediatric patients: 10 years of experience in a single centre
}

\author{
Mario Lima, Niel Di Salvo, Giovanni Marchi, Vincenzo Davide Catania, Michele Libri, Tommaso Gargano \\ Department of Pediatric Surgery, Sant'Orsola University-Hospital, University of Bologna, Italy
}

\begin{abstract}
Peritoneal Dialysis (PD) is one of the numerous options for chronic dialysis and in many cases when access for acute dialysis is required early in a hospital course, at any age. PD catheter can be inserted with an open or laparoscopic approach. The complication rate after catheter insertion is still high, as reported in published literature. We present the experience matured at our Centre in the last 10 years on implantation of peritoneal dialysis catheters in children, emphasising surgical complications. We conducted a retrospective study on patients who underwent PD at our Centre in a range period of 10 years. We analysed patients 'demographic data, past and present medical, perioperative and post-operative data, permanence of the catheter, duration of dialysis, the gap between placement and use, outcomes and complications. We compared the data, dividing patients in 2 groups: patients operated with a traditional open technique and patients operated laparoscopically. We retrospectively reviewed 29 children with an average age of $3 y e a r s$ and 6 months. Mean age was 42 months (1 month; 8 years) for the VLS group, 18 months (11 days, 4 years) for the OT group. Mean operative time was 106 min for the VLS group; 44 min for the OT group. The Catheter permanence period was 17 days (12h-64 days). Duration of dialysis was between 48 hours and 23 days (average 8 days). In the
\end{abstract}

\footnotetext{
Correspondence: Niel Di Salvo, Department of Pediatric Surgery, Sant'Orsola University-Hospital, University of Bologna, via G.

Massarenti 11, 40138 Bologna, Italy.

E-mail: neildisalvo@hotmail.com

Tel.: +393889066813.

Key words: Peritoneal dialysis catheters; Pediatric renal replacement therapy; Tenchkoff; Peritoneal dialysis; Complications during peritoneal dialysis.

Conflict of interest: the authors declare conflict of interests.

Received for publication: 11 October 2019

Accepted for publication: 14 August 2020.

This work is licensed under a Creative Commons Attribution

NonCommercial 4.0 License (CC BY-NC 4.0).

OCopyright: the Author(s), 2020

Licensee PAGEPress, Italy

La Pediatria Medica e Chirurgica 2020; 42:221

doi:10.4081/pmc.2020.221
}

total population, we registered 8 complications (5 minor, 3 major), the overall complication rate being $33 \%$ (minor complication rate $21 \%$, major complication rate $12,5 \%$ ). 6 complications occurred in patients operated laparoscopically $(6 / 14=36$ $\%) ; 2$ complications in the OT group $(2 / 10=20 \%)$. The complication rate after PD catheter insertion is still high. Advantages and disadvantages of the open and laparoscopic approach must be known. Both minor and major complications, such as bowel perforations and occlusions, must be understood and differentiated.

\section{Introduction}

Peritoneal Dialysis (PD) is one of the numerous options for chronic dialysis. PD and intravenous catheter-based dialysis are often performed in many cases when access for acute dialysis is required early in a hospital course. Age is not a limiting factor for PD; in fact, peritoneal catheters have successfully been placed in newborns as young as a few days old. In patients of adequate size and in whom adhesions do not present sufficient risk, a laparoscopic approach may be considered. ${ }^{1}$ Laparoscopy was initially used in adults to rescue blocked PD catheters and, later, for initial PD catheter placement. By facilitating a thorough exploration of the abdominal cavity, permitting identification of coincidental intra-abdominal disease and careful placement of the PD catheter tip, laparoscopy has been suggested to promote longer catheter survival than open placement. However, the optimal placement technique remains uncertain. Placing PD catheters in children poses unique challenges reflected in the high complication rates for children, as high as $70 \%$ in some series. ${ }^{2}$ The most common complications associated with PD continue to be catheter infections and peritonitis. Complications such as obstruction, non-functioning or malpositioned catheters, and leakage occur commonly in children.1,3 Recently, many modifications have been proposed in order to reduce complications related to peritoneal dialysis and to PD catheter placement as: downward and lateral exits sites, and various dressings and treatments to reduce catheter site infection and/or peritonitis. ${ }^{3-6}$ Furthermore, omentectomy have been advocated to reduce the rate of catheter obstruction. Recently, an experience with laparoscopic PD catheter placement has been evaluated to determine the impact on complications. ${ }^{4}$ At our institution, open PD catheter placement and laparoscopic-assisted PD catheter placement have been used based upon individual surgeon preference, or patient's conditions.

We describe the experience matured at our Centre in the last 10 years on implantation of peritoneal dialysis catheters in children, emphasising surgical complications. 


\section{Materials and Methods}

We conducted a retrospective study on patients who underwent PD at our Centre in a range period of 10 years (between 2008 and 2018). At Our Centre, we use double cuff straight peritoneal dialysis catheter (Tenchkoff) with the exception of neonates on whom a 1 subcutaneous cuff straight peritoneal dialysis is applied. PD catheters are positioned with an open or laparoscopic technique. The technique adopted was chosen based on anatomic conditions, age, general clinical conditions and surgeon's preference.

We analysed patients 'demographic data, past and present medical history including etiology of renal insufficiency, perioperative and post-operative data such as type of surgical approach (conventional vs laparoscopic), operative time, permanence of the catheter, duration of dialysis, the gap between placement and use, outcomes and complications. The minimum follow-up required to be included in the analysis was 1 year. We therefore excluded patients of whom we could not gain the above-mentioned information.

These data were extrapolated from medical charts, Operative Room register and outpatient reports; we compared the data, dividing patients in 2 groups: patients operated with a traditional Open Technique (OT) and patients operated laparoscopically (VLS).

\section{Results}

On 14 patients (63\%) we used a laparoscopic approach (VLS), 10 patients $(32 \%)$ were instead operated with an open conventional technique

In the study period (10 years, between 2008 and 2018), we retrospectively reviewed 29 children in an overall age range between 11 days and 8 years (average 3years and 6 months) that underwent surgery for the implant of a Tenchkoff catheter.

Among our total population of 29 patients, 5 were excluded for

\section{Table 1. Patients' demographics.}

\begin{tabular}{ll}
\hline Number of patients & 29 \\
Sex & F $12(49 \%)$ \\
& M $17(51 \%)$ \\
\hline Age at surgery & 3 years 6 months [11 days-8 years] \\
Etiology of ARK & i) 27 HUS \\
& ii) 1 urethral atresia \\
& iii) 1 vasculitis \\
\hline
\end{tabular}

Table 2. Results.

\begin{tabular}{ll}
\hline $\begin{array}{l}\text { Catheter permanence period } \\
\text { Duration of dialysis }\end{array}$ & 17 days [12 hours-64 days] \\
\hline Surgical approach & Open $10(37 \%$ hours-23 days] \\
& VLS $14(63 \%)$ \\
Operative time & Open 44 min \\
& VLS 106 min \\
\hline Complications & i) 3 catheter obstructions \\
& ii) 1 leakage \\
& iii) lintestinal obstruction \\
& iv) 2 ileo-colic perforations \\
& v) 1 bacterial peritonitis \\
& vi) 1 hydrocele \\
\hline
\end{tabular}

death of patients (2), related to the pathology, and lack of information in regard to long term follow-up ( 3 patients lost on follow-up).

Patients' demographics are summarized in Table 1; results are summarized in Table 2.

It must be noticed from Table 2 how the majority of Acute Renal Injury (AKI) was due to Hemolytic-Uremic Syndrome (HUS). On 14 patients (63\%) we used a laparoscopic approach (VLS), 10 patients (32\%) were instead operated with an open conventional technique. Mean age was 42 months ( 1 month; 8 years) for the VLS group, 18 months (11 days, 4 years) for the OT group. Mean operative time was $106 \mathrm{~min}$ for the VLS group; $44 \mathrm{~min}$ for the OT group. The Catheter permanence period was 17 days (12h64 days). Duration of dialysis was between 48 hours and 23 days (average 8 days).

In the total population, we registered 8 complications ( 5 minor, 3 major), the overall complication rate being $33 \%$ (minor complication rate $21 \%$, major complication rate $12,5 \%$ ). 6 complications occurred in patients operated laparoscopically $(6 / 14=36 \%) ; 2$ complications in the OT group $(2 / 10=20 \%)$.

Regarding minor complications, we registered 2 cases of outflow failure, 1 case of inflow problems, 1 of leakage during PD, 1 peritonitis secondary to catheter infection, 1 case of hypertensive hydrocele. In all cases of flow impairment PD had to be suspended and a second surgical procedure was necessary.

In the three cases of outflow impairment, the catheters resulted obstructed by remnant portions of the omentum; in the only case of inflow problems the catheter resulted dislodged. In the leakage patient, a conservative management was successfully instituted.

In another patient, operated laparoscopically, the formation of a huge hypertensive hydrocele for patent processus vaginalis during PD did not allow PD to be completed; the patient had to be reoperated for closure of patent processus vaginalis.

In our case series, we registered 3 major intestinal complications: 2 bowel perforations, 1 bowl occlusion.

The first case regards a 3 -year old female who, during the $17^{\text {th }}$ day of PD for acute renal failure secondary to HUS, presented a worsening of her general clinical conditions, acute abdomen and sepsis. Emergency laparotomy revealed a single punctiform perforation of the descending colon managed with descending colon resection and protective colostomy (Figure 1).

A Different case regarded a 2-year old male who, during PD for

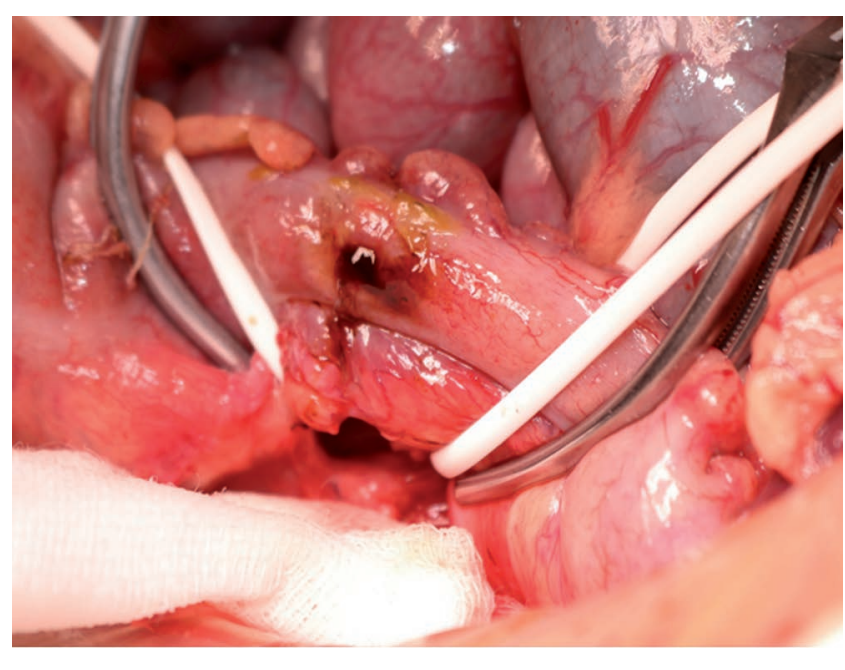

Figure 1. Punctiform perforation of the descending colon (decubitus delayed bowel perforation). 
acute renal failure secondary to HUS, presented an acute abdomen on 10th Day of PD; emergency laparotomy showed multiple colic perforations that comprehended caecum, ascending transverse and descending colon; besides all the perforated segments, the colic walls looked edematous and fragile with petechiae; the patient was operated with the creation of protective ileostomy (Figure 2).

These two cases, even though they look similar, are different from a pathogenetic standpoint. The first perforation was in fact likely to be correlated to the tube itself (decubitus damage). In the second case, being a more diffuse reaction condition of the colon, was probably due to diffusion of the HUS to the colon.

A 3-year old male, already known for $\mathrm{CDH}$ corrected with a laparotomic approach in the neonatal period, developed a bowel occlusion a month after removal of a Tenchkoff catheter used for PD in kidney failure secondary to HUS. During laparotomy, the occlusion was located on the distal ileum, covered by omentum under which a micro-perforation was detected. He was treated with protective ileostomy (Figure 3).

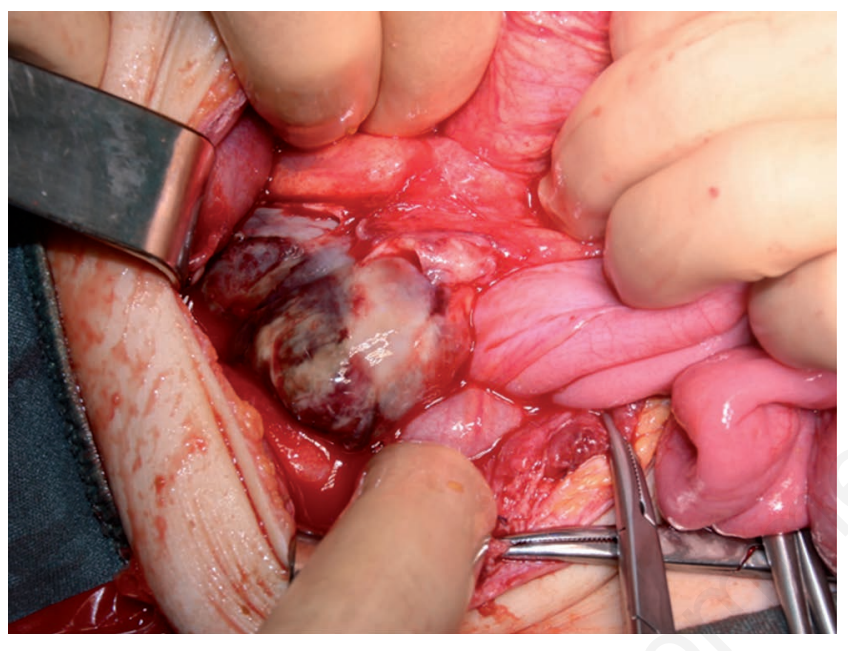

Figure 2. Full-thickness colitis with multiple colic perforation of a patient with $\mathrm{HU}$.

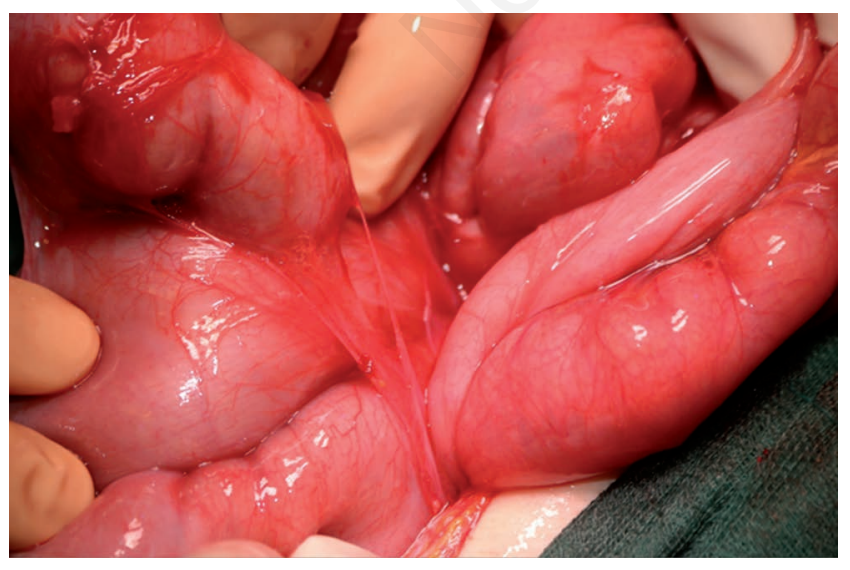

Figure 3. Bowel occlusion determined by peritoneal adherence in a patient who underwent PD through a Tenchkoff catheter removed a month before the onset of acute abdomen. Under the adherence, a microperforation, perhaps related to decubitus of the catheter, was detected.

\section{Discussion}

Peritoneal Dialysis (PD) is one of the numerous options for chronic dialysis. PD and intravenous catheter-based dialysis are often performed in many cases when access for acute dialysis is required early in a hospital course. Age is not a limiting factor for PD; in fact, peritoneal catheters have successfully been placed in new-borns as young as a few days old. ${ }^{1}$ Laparoscopic surgery has become increasingly popular for PD catheter placement. We described the experience matured at our Centre in the last 10 years on implantation of peritoneal dialysis catheters in children, emphasising surgical complications. The complication rate after catheter insertion is still high, as reported in published literature. In our case series, we registered an overall complication rate of $33 \%$ (minor complication rate $21 \%$, major complication rate $12,5 \%$ ). The laparoscopic technique of insertion seems to increase operative times (VLS 106 minutes vs. OT $44 \mathrm{~min}$ ), confirmed in literature, and to be associated to a slightly higher complication rate (VLS 36 $\%$; OT $2 / 10=20 \%$ ). Literature comparing laparoscopic to open PD catheter placement is limited and there is no consensus regarding an optimal technique. In fact, reports have failed to demonstrate a technique that results in decreased early complication rates in children. ${ }^{3}$ Carpenter was able to demonstrate that late catheter complication rates appeared to be higher with open surgical placements as well as the rates of re-operation; nevertheless, this may reflect a bias with younger, more critically ill, patients preferentially undergoing an open procedure. ${ }^{3}$

Laparoscopy gives the chance to close an eventual patent processus vaginalis, which can be problematic during PD. As suggested by the study of Sodo et al. 5 in the adult population, simultaneous surgical abdominal wall defect repair and peritoneal catheter insertion can be regarded as a safe surgical procedure. This strategy makes PD possible for some patients who would otherwise be excluded from the possibility of PD and, in addition, it eliminates the risks of repeated anaesthesia and reduces costs of hospitalization.

Common complications include outflow failure, for omentum or remnant portions of the omentum obstructing the catheter, inflow problems, likely due to malposition, and leakage. The first two complications usually require a second surgical procedure. If the patient's situation allows, it would be ideal not to use the catheter for 4 weeks in order to avoid leakage which is a very common complication when necessary to perform PD immediately after placement. This can be treated by decreasing the amount of dialysate and increasing the number of exchanges. Nearly all these leaks will seal with conservative management unless the inner cuff has been dislodged. Others have described common complications as being post-operative intra-abdominal bleeding and peritonitis/catheter infection. Delayed bowel perforation of a PD catheter is an uncommon complication. Perforative peritonitis in a patient undergoing PD is often difficult to differentiate from the more common peritonitis secondary to catheter infection because free air and ascites are not specific. In fact, air is often introduced in the abdominal cavity with dialysate infusion due to recurrent technique error.

Bowel perforations can occur during acute and chronic dialysis. Decubitus bowel perforations have to be differentiated from HUS bowel perforations

Delayed bowel perforations in patients on chronic dialysis without associated intestinal conditions have been described as isolated case reports. ${ }^{6}$ A long duration of a PD catheter in the abdominal cavity without peritoneal fluid, which bathes the bowel loops acting as a barrier of adhesion of the catheter to the bowel 
wall, increases the risk of pressure-induced necrosis by the immobile catheter. The mechanism of perforation has also been debated in these above-mentioned case reports. Some authors suggest that a delayed perforation is the result of continuous pressure necrosis from the catheter tip on the bowel wall initiated by the unused peritoneal catheter due to the SMAP and fixing the catheter tip due to the PWAT. ${ }^{3}$ According to others, instead, when the lower cuff of the double-cuffed catheters migrates into the peritoneal cavity, adhesion of the cuff to the intestinal wall may be another mechanism of bowel perforation. ${ }^{7}$

\section{Conclusions}

Peritoneal Dialysis (PD) is one of the numerous options for chronic dialysis. PD and intravenous catheter-based dialysis are often performed in many cases when access for acute dialysis is required early in a hospital course. Age is not a limiting factor for PD; in fact, peritoneal catheters have successfully been placed in new-borns as young as a few days old. In patients of adequate size and in whom adhesions do not present sufficient risk, a laparoscopic approach may be considered. Advantages and disadvantages of each approach must be known. Complication rates are still high. The major, abdominal ones, such as bowel perforations and occlusions, must be understood and differentiated.

\section{References}

1. Cribbs RK, Greenbaum LA, Heiss KF. Risk factors for early peritoneal dialysis catheter failure in children. J Pediatr Surg 2010;45:585-9. doi: 10.1016/j.jpedsurg.2009.06.019.

2. JarBoe M and hirschl RB. Ch. 89 "Dialysis" in Operative Pediatric Surgery, 7th edition, Coran, Spitz. CRC PRESS; 2013. Pp. 862-871.

3. Carpenter JL, Fallon SC, Swartz SJ, et al. Outcomes after peritoneal dialysis catheter placement. J Pediatr Surg. 2016;51:730-3. doi: 10.1016/j.jpedsurg.2016.02.011.

4. LaPlant MB, Saltzman DA, Segura BJ, et al. Peritoneal dialysis catheter placement, outcomes and complications. Pediatr Surg Int 2018;34:1239-44. doi: 10.1007/s00383-018-4342-1.

5. Sodo M, Bracale U, Argentino G, et al. Simultaneous abdominal wall defect repair and Tenckhoff catheter placement in candidates for peritoneal dialysis. J Nephrol 2016;29:699-702. doi: 10.1007/s40620-015-0251-8

6. Fujiwara M, Soda T, Okada T, et al. Bowel perforation by a peritoneal dialysis catheter: report of two cases. BMC Nephrology 2017;18:312. doi: 10.1186/s12882-017-0737-9

7. Ratajczak A, Lange-Ratajczak M, Bobkiewicz A, Studniarek A. Surgical management if complications with peritoneal dialysis. Semin Dialysis 2017;30:63-8. doi: 10.1111/sdi.12538. 